\title{
A factor analysis of data from sequential alternation of amygdaloid stimulation: A replication
}

\author{
JOHN GAITO and STEPHEN T. GAITO \\ York University, 4700 Keele Street, Downsview, Ontario, M3J 1P3 Canada
}

\begin{abstract}
Data from a number of sequential alternation experiments for 125 subjects were factor analyzed to determine the number of common factors present. Three measures (mean latency of convulsion, mean number of trials to, six convulsions, mean duration of convulsions) were evaluated by principal components analyses. The presence of two factors was suggested in the latency, criterion, and duration measures (primary site stimulation, secondary site stimulation). The two factors were more clearly defined for the latency data than for the other measures. Further analyses with individual trials (rather than means) for the 125 subjects provided approximately the same results. Factor analyses of data from 35 rats stimulated only on one side showed the presence of one factor in all analyses. These results suggest a two-factor interpretation of kindling events, possibly the two effects of Goddard et al. and McIntyre and Goddard: a long-term neurological circuitry modification for each of the primary and secondary sites and a short-term aftereffect which accounts for the negativetransfer aspects from the primary to the secondary site.
\end{abstract}

The "kindling effect" has been investigated in a number of laboratories as a model of learning, a model of epilepsy, or as an example of behavioral change of interest in its own right (e.g., Gaito \& Gaito, 1974; Goddard, McIntyre, \& Leech, 1969; Racine, 1972; Wada \& Sato, 1975). The effect consists of a progressive change in the overt behavior of the animal, from normal exploration (Stage $1-\mathrm{NE}$ ), to behavioral automatisms (Stage 2-BA; chewing, eye closure on ipsilateral side, salivation), and finally to clonic convulsions (Stage 3-CC) in response to electrical stimulation of a specific brain site (e.g., amygdala). Stage 3 behavior involves the rat standing on its hind paws, and bilateral convulsions of the forelimbs occur. Behavioral, chemical, electrophysiological, and neurological aspects of this effect have been investigated by many researchers (Gaito, 1976a; Racine, 1978).

We have conducted a number of experiments in which rats were stimulated alternately in each amygdala to a specific number of convulsions (e.g., Gaito, 1976b). First, a rat is stimulated on one side (primary site) until 6 convulsions occur. Then stimulation is shifted to the contralateral side (secondary site) for 6 convulsions. Then the primary site is stimulated a second time, followed by stimulation of the secondary site, etc. This sequential alternation of stimulation continues for 10 phases of 6 convulsions each, or 60 convulsions overall.

In previous papers, we have described factor analyses concerned with determining the number of common factors underlying data involving phase means (Gaito, Gaito, \& Nobrega, 1977) and also data involving each of the 60 convulsion trials (Gaito \& Gaito, 1979). The results indicated two clearly separated factors in both cases with latency data (time between onset of stimulation and onset of convulsion): the primary site stimulation and the secondary site stimulation factor. In phase data, the criterion means (number of trials to achieve 6 convulsions) also indicated the same two factors. The duration measure (time between onset and offset of convulsion) showed one factor for phase means data but two when the 60 trials were analyzed. However, the two factors were not as clear as with the latency analyses. In the experiments from which these data were obtained, the same intensity of stimulation was used with each side.

In the present paper, we report factor analyses similar to the previous ones. However, the data used in these analyses were from rats that were stimulated on each side with an intensity that was just above threshold (e.g., Gaito, 1977). The purpose of these analyses was threefold: (1) to check on the replicability of the previous results using data from the threshold stimulation method; (2) to contrast twoside-stimulation data with that of one-side stimulation; and (3) to determine the number of common factors present so as to relate these results to possible neurological functioning.

\section{METHOD}

The data from previous experiments were used. In each experiment, male Wistar rats were implanted bilaterally in the amygdala with bipolar electrodes (Nichrome wire with trimel coating, $.127 \mathrm{~mm}$ in diameter). The brain coordinates for elec- 
Table 1

Means and Standard Deviations for the 10 Phases

\begin{tabular}{|c|c|c|c|c|c|c|c|c|c|c|}
\hline & \multicolumn{10}{|c|}{ Phase } \\
\hline & 1 & 2 & 3 & 4 & 5 & 6 & 7 & 8 & 9 & 10 \\
\hline & \multicolumn{10}{|c|}{ Latency } \\
\hline Mean & 8.8 & 11.1 & 7.1 & 9.6 & 7.4 & 9.9 & 7.9 & 10.1 & 8.1 & 10.4 \\
\hline \multirow[t]{2}{*}{ SD } & 6.5 & 7.7 & 4.5 & 7.1 & 4.6 & 7.4 & 5.0 & 6.6 & 5.4 & 6.7 \\
\hline & \multicolumn{10}{|c|}{ Criterion } \\
\hline Mean & 25.4 & 21.7 & 7.0 & 7.1 & 6.9 & 6.6 & 6.8 & 6.6 & 6.8 & 6.6 \\
\hline \multirow{2}{*}{ SD } & 13.6 & 10.7 & 2.7 & 1.9 & 1.5 & 1.1 & 1.6 & 1.3 & 1.5 & 1.3 \\
\hline & \multicolumn{10}{|c|}{ Duration } \\
\hline Mean & 20.8 & 22.4 & 23.5 & 24.1 & 25.0 & 24.0 & 24.2 & 24.3 & 23.2 & 24.3 \\
\hline SD & 5.5 & 6.0 & 6.6 & 6.3 & 8.1 & 7.9 & 7.9 & 7.9 & 8.6 & 7.8 \\
\hline
\end{tabular}

Note $-N=125$.

trode implantation were the same in all experiments: $.5 \mathrm{~mm}$ posterior to bregma, $4.5 \mathrm{~mm}$ from midline, $8.5 \mathrm{~mm}$ from skull (Gaito \& Gaito, 1974). Seven or more days after surgery, brain stimulation began. Each trial consisted of passing a $60-\mathrm{Hz}$ sine wave for $30 \mathrm{sec}$ into the rat using a Lafayette stimulator; there were three trials per day with approximately $1 \mathrm{~h}$ between trials. The initial intensity was $100 \mu \mathrm{A}$ for all animals; in the cases of no clonic convulsion (CC) by 10 trials, the current was gradually increased until the appropriate intensity was achieved for each animal. In most cases, the convulsion was achieved with $100 \mu \mathrm{A}$, and in no case was the intensity level raised beyond $560 \mu \mathrm{A}$.

After six convulsions were achieved at the primary site (Phase 1), a similar procedure was followed with the secondary site in Phase 2 until six convulsions occurred. This alternation from primary to secondary site continued for 10 phases.

On the first trial of Phases 3 and 4, the intensity was slowly increased from subthreshold values until a definite response occurred. Then, in order to accommodate day-to-day fluctuations, the current was increased approximately $15 \mu \mathrm{A}$ beyond this point.

The data used for the alternation study were from 125 rats, which were between 120 and 200 days of age at the beginning of the experiment. Analyses were completed also on 35 rats which were stimulated only on one side. The data for 10 phases $(60$ CC trials) with three dependent variables were analyzed: latency (time from onset of stimulation to onset of convulsion), criterion (number of trials to $6 \mathrm{CC}$ ), and duration of convulsion (time from onset of forelimb convulsion to complete cessation of convulsion).

For each rat, the behavioral designations for the latency, duration, and criterion measures were primary oscillator, secondary oscillator, or nonoscillator. For example, to be classified as an oscillator, the rat had to show a consistent pattern of "low-high" (primary oscillation) or "high-low" (secondary oscillation) in latency, duration, and/or criterion data over 8 or 10 of the 10 alternation phases.

In the previous studies, we used a principal components analysis. This procedure allows for $\mathbf{k}$ factors to be extracted if $\mathbf{k}$ variables are involved. However, each successive factor extracts maximum variance and many of the later $k$ factors are trivial. The scree test and characteristic root $\geqslant 1$ were used as criteria to determine the number of significant factors present. These criteria provide better results than that using a chi-square test of correlation matrix residuals after each factor extraction; this latter procedure tends to overestimate the number of factors (Gorsuch, 1974).

Principal components anaylses were performed for each of the three dependent variables in phase data, and for latency and duration measures in trial data. The criterion measure is lost when individual trials are analyzed. Product moment correlation coefficients were obtained over the 10 phases and over the 60 trials for the factor analyses. All computatons were obtained using the Statistical Package for the Social Sciences, SPSS-10, the University of Pittsburgh, on IBM 370 and DEC system-10 computers at York University.

\section{RESULTS}

\section{Phase Data}

Means, standard deviations. The means and standard deviations for the 125 subjects are shown in Table 1. The patterns were similar to the results reported in the previous paper for 139 subjects (Gaito et al., 1977). The means for latency followed a "low-high" pattern over the 10 phases, with the mean values for the primary site being lower than that for the following secondary site for all phases, resulting in a saw-tooth oscillation type curve. ${ }^{1}$ In criterion data, means and standard deviations for Phases 1 and 2 were the greatest and a sharp drop occurred in Phase 3. Phases 3 to 10 had approximately the same mean. ${ }^{1}$ The duration means showed an opposite pattern to the criterion measure; the first phase had the smallest mean, and Phases 2 to 10 showed approximately the same mean values. ${ }^{1}$ Relative stability was attained in Phase 2 with duration data and in Phases 3 and 4 with the other measures.

The standard deviations for the latency and duration measures were relatively constant over the 10 phases. However, in criterion data, the variability was sizable during Phases 1 and 2 but diminished sharply thereafter.

Intercorrelations. ${ }^{2}$ In latency data, the coefficients were all positive. The correlation between Phases 1 and 2 was low, indicating little relationship between behavior in Phase 1 and in Phase 2. Also, there was the tendency for the correlation between phases on the same side (e.g., 1-3, 1-5, 2-4, 2-6, etc.) to be higher than that between phases on different sides. This tendency involves an alternation of values ("low-high") in the table.

In criterion data, both positive and negative coefficients occurred. The correlation between Phases 1 and 2 was essentially zero. Again, there was the pattern in which correlations for the same-side phases were different from those for the different-side phases. For example, the relationship of odd-to-odd numbers and of even-to-even numbers was of a positive nature, whereas the other relationships 
(odd-even and even-odd) tended to be of negative nature. Also, the correlation of Phase 1 and other odd phases and of Phase 2 and other even phases tended to be lower than the correlation between similar phases after Phase 2. This result suggested greater stability in this behavioral variable beginning with Phase 3. This aspect is reflected also in Table 1, where the standard deviations tended to be greater for Phases 1 and 2 than for later phases.

In the duration measure, the coefficients were all positive. It is interesting to note that the correlations tended to be more homogeneous, especially for adjacent phases, than did those for the latency and criterion data wherein an alternating pattern of "low-high" or "positive-negative" occurred for a series of correlations.

The value for $r$ required to reject the null hypothesis of zero correlation for 125 subjects is $.176(.05$ probability level). In the criterion measure, fewer correlation coefficients were significantly different from zero than was the case with latency and duration data.

Factor analyses. ${ }^{2}$ In these analyses, two criteria were used to differentiate significant factors from trivial ones: characteristic roots $\geqslant 1$ and the scree test (Gorsuch, 1974). The latter test involves plotting the characteristic roots or eigenvalues for the 10 phases. The characteristic roots for trivial factors are in a straight line, whereas those for significant factors show a sharp break or cliff effect.

Factor extraction provides an ambiguous solution because the axes are arbitrary. Thus, an orthogonal rotation procedure (Equimax) was used to provide a solution easy to interpret with the three measures. The original factor loadings were transformed (via a transformation matrix) to the final factor loadings.

With latency data, two factors emerged according to both the scree test and characteristic roots criteria. More than $80 \%$ of the variance was accounted for by the two factors. The orthogonal rotation procedure provided a clear separation of the two factors. There were high loadings on one factor and low ones on the other. Phase 1 had the lowest communality, ${ }^{3}$ approximately .500 . The two factors were the same as in the previous analyses: the primary site stimulation factor and the secondary site stimulation factor. The angle between the cluster of phases on each of the two factors was approximately $70 \mathrm{deg}$, indicating low correlation between them, a result also similar to that obtained in the previous analyses.

The factor analysis results with the criterion and duration measures were not as clear as with the latency data. The intercorrelation matrix for the criterion measure contained many nonsignificant rs. Thus, it is not surprising that the scree test suggested two factors (accounting for $49.5 \%$ of the variance) whereas the characteristic roots criterion suggested three factors (61.6\% of variance). Two- and threefactor rotations were provided. The two-factor rotation provided a solution easy to interpret, and the three-factor resolution did not improve on this aspect.

The duration results were cloudy. The scree test suggested one factor (accounting for about 50\% of variance), whereas the characteristic roots criterion indicated three factors. One-factor extraction, a general factor, would be suitable and is the same as that observed in the previous analyses. If a twofactor resolution is used, the plot of these factors indicates a pattern lacking clarity. Like the criterion results, Phases 1 and 2 did not cluster with their respective phases of the same side.

The communalities for the various phases for both criterion and duration data were much lower than was the case with latency measure. The lowest values occurred in the criterion measure, especially in Phase 1.

\section{Individual Trial Data}

Means, standard deviations, linear regression coefficients. The means and standard deviations over the 60 trials for the 125 subjects are presented in Tables 2 and 3 for the latency and duration measures.

Table 2

Means (M) and Standard Deviations (SD) for the 60 Trials (T) and the Linear Regression Coefficients (b) for Each Set of 6 Trials for the Latency Measure

\begin{tabular}{|c|c|c|c|c|c|c|c|}
\hline $\mathrm{T}$ & $\mathbf{M}$ & $\mathrm{SD}$ & $b$ & $\mathrm{~T}$ & $\mathbf{M}$ & SD & $\mathrm{b}$ \\
\hline 1 & 13.9 & 10.1 & & 31 & 10.9 & 9.7 & \\
\hline 2 & 10.4 & 7.8 & & 32 & 9.8 & 7.7 & \\
\hline 3 & 8.7 & 7.1 & & 33 & 10.3 & 8.4 & \\
\hline 4 & 7.5 & 7.0 & & 34 & 9.9 & 7.7 & \\
\hline 5 & 6.7 & 6.0 & & 35 & 9.7 & 7.2 & \\
\hline 6 & 6.0 & 6.1 & -1.48 & 36 & 9.5 & 6.8 & -.22 \\
\hline 7 & 14.7 & 9.4 & & 37 & 9.2 & 6.5 & \\
\hline 8 & 11.9 & 8.1 & & 38 & 7.6 & 5.5 & \\
\hline 9 & 11.1 & 8.6 & & 39 & 8.0 & 5.7 & \\
\hline 10 & 9.9 & 8.3 & & 40 & 7.7 & 5.4 & \\
\hline 11 & 9.9 & 8.8 & & 41 & 7.4 & 5.2 & \\
\hline 12 & 9.5 & 8.0 & -.95 & 42 & 7.6 & 5.2 & -.25 \\
\hline 13 & 9.0 & 7.4 & & 43 & 11.4 & 8.8 & \\
\hline 14 & 7.2 & 5.6 & & 44 & 9.7 & 7.3 & \\
\hline 15 & 7.3 & 5.2 & & 45 & 10.2 & 7.4 & \\
\hline 16 & 6.7 & 4.5 & & 46 & 10.1 & 6.7 & \\
\hline 17 & 6.2 & 4.2 & & 47 & 9.6 & 6.4 & \\
\hline 18 & 6.6 & 4.3 & -.45 & 48 & 9.4 & 6.5 & -.30 \\
\hline 19 & 12.2 & 9.1 & & 49 & 9.0 & 6.5 & \\
\hline 20 & 9.5 & 7.8 & & 50 & 8.1 & 6.2 & \\
\hline 21 & 9.9 & 8.2 & & 51 & 8.4 & 5.8 & \\
\hline 22 & 8.9 & 7.1 & & 52 & 7.8 & 5.6 & \\
\hline 23 & 8.5 & 7.2 & & 53 & 7.7 & 5.6 & \\
\hline 24 & 9.0 & 7.4 & -.57 & 54 & 7.8 & 5.3 & -.22 \\
\hline 25 & 8.1 & 5.8 & & 55 & 11.8 & 9.1 & \\
\hline 26 & 7.3 & 5.0 & & 56 & 10.6 & 7.7 & \\
\hline 27 & 7.6 & 5.0 & & 57 & 10.3 & 7.0 & \\
\hline 28 & 7.2 & 4.8 & & 58 & 10.3 & 7.1 & \\
\hline 29 & 7.2 & 4.9 & & 59 & 9.8 & 6.5 & \\
\hline 30 & 7.2 & 5.0 & -.15 & 60 & 9.7 & 6.4 & -.37 \\
\hline
\end{tabular}


Table 3

Means (M) and Standard Deviations (SD) for the 60 Trials and the Linear Regression Coefficients (b) for Each Set of 6 Trials for the Duration Measure

\begin{tabular}{rrrrrrrr}
\hline $\mathrm{T}$ & $\mathrm{M}$ & $\mathrm{SD}$ & $\mathrm{b}$ & $\mathrm{T}$ & $\mathrm{M}$ & $\mathrm{SD}$ & $\mathrm{b}$ \\
\hline 1 & 14.5 & 6.0 & & 31 & 22.2 & 10.8 & \\
2 & 19.2 & 7.5 & & 32 & 23.9 & 10.4 & \\
3 & 21.9 & 9.2 & & 33 & 24.7 & 10.4 & \\
4 & 22.5 & 8.8 & & 34 & 24.7 & 10.4 & \\
5 & 22.9 & 7.3 & & 35 & 24.4 & 9.6 & \\
6 & 23.8 & 8.2 & 1.66 & 36 & 24.2 & 9.0 & .33 \\
7 & 19.6 & 9.9 & & 37 & 22.6 & 12.9 & \\
8 & 21.8 & 8.3 & & 38 & 24.5 & 10.5 & \\
9 & 22.3 & 9.0 & & 39 & 25.2 & 10.5 & \\
10 & 23.3 & 8.7 & & 40 & 24.5 & 10.0 & \\
11 & 23.5 & 8.4 & & 41 & 24.0 & 9.7 & \\
12 & 24.1 & 7.5 & .82 & 42 & 24.5 & 9.2 & .21 \\
13 & 21.1 & 9.1 & & 43 & 23.3 & 11.5 & \\
14 & 24.5 & 9.9 & & 44 & 25.0 & 10.4 & \\
15 & 24.4 & 10.5 & & 45 & 26.4 & 10.4 & \\
16 & 23.2 & 8.8 & & 46 & 23.6 & 10.6 & \\
17 & 23.5 & 8.3 & & 47 & 23.7 & 9.7 & \\
18 & 24.6 & 8.3 & .38 & 48 & 23.7 & 8.9 & .13 \\
19 & 22.1 & 9.2 & & 49 & 21.5 & 12.4 & \\
20 & 24.2 & 9.2 & & 50 & 24.3 & 11.2 & \\
21 & 23.9 & 9.5 & & 51 & 24.1 & 11.0 & \\
22 & 24.2 & 9.2 & & 52 & 23.2 & 9.9 & \\
23 & 25.5 & 7.8 & & 53 & 24.3 & 10.5 & \\
24 & 24.9 & 8.2 & .52 & 54 & 23.4 & 9.4 & .25 \\
25 & 22.9 & 12.2 & & 55 & 22.3 & 11.5 & \\
26 & 24.6 & 12.2 & & 56 & 25.5 & 10.6 & \\
27 & 25.1 & 11.1 & & 57 & 25.0 & 10.7 & \\
28 & 25.1 & 10.8 & & 58 & 25.0 & 11.0 & \\
29 & 24.0 & 9.2 & & 59 & 24.4 & 9.6 & \\
30 & 26.0 & 8.9 & .39 & 60 & 23.7 & 9.6 & .11 \\
\hline
\end{tabular}

These tables contain also the linear regression coefficients for each set of six trials in a phase over the 60 trials.

The linear regression coefficients indicate that at first the trend of the two measures were in opposite directions. Latency means during the first 12 trials (Phases 1 and 2) indicated a sharp decrement over trials, whereas the duration means showed a sharp increment. Thereafter there was a more gradual change. By Phases 3 and 4 (Trials 13-24), the pattern for latency had become relatively stable; this aspect was indicated clearly also when the means were analyzed previously. Relative stability for the duration measure was achieved by Phase 2 .

For the latency data, the means for the secondary site trials $(7-12,19-24$, etc.) were greater than those for the primary site $(1-6,13-18$, etc.) over the entire 60 trials. Also, the sets of six primary site trials tended to have lower regression coefficients after the first two phases.

The first set of six trials was the most variable.

Intercorrelations. ${ }^{2}$ The intercorrelations between the 60 trials were computed. The important results for latency data were: (1) Coefficients of Trial 1 with each of the other trials in phases other than Phase 1 tended to be the lowest. (2) By Phase 2, the $r$ values for between trials within the same phase were stable at approximately .60 or greater. (3) The $r$ values for between trials of opposite-side phases were the lowest and seldom went above .40. However, if the trials of Phase 1 are excluded, these $r$ values are of positive nature and tend to be significant ones. This aspect is probably indicating positive transfer over opposite phases.

The results for the duration measure were: (1) The highest rs occurred between adjacent trials, even when one trial involved the primary site and the other, the secondary site. This result is in sharp contrast to the latency measure under these conditions wherein the lowest rs occurred; for the latency data, there were sharp breaks between each phase of six trials, with high rs in one phase alternating with low rs in the next phase. (2) Highest rs occurred also between trials within the sets of six for each phase. (3) None of the rs were greater than about .60. This result is also in sharp contrast to latency data where rs of .60 to .80 were common. (4) About half of the rs were nonsignificant ones, or just barely above the .176 required to reject the null hypothesis of zero correlation at a p level of .05 with 125 subjects. More significant rs occurred within latency data.

Factor analyses. ${ }^{2}$ For latency data, the scree test suggested two factors whereas the characteristic roots criterion indicated up to five. However, rotation procedures with two to five factors indicated that a two-factor solution provided the clearest picture. These factors were the primary site stimulation factor and the secondary site stimulation factor. The two-factor extraction accounted for $70.1 \%$ of the variance. Five-factor extraction would have added only $10.6 \%$ more of the variance.

With the two-factor solution, the lowest communality value was for Trial 1 . The communalities for the remaining trials were moderate to high.

The plot of the two-factor solution showed a clear separation of the two factors with an angle of approximately $70 \mathrm{deg}$ between them. This result is consistent with previous latency results and indicates a low correlation between the factors for the primary and secondary sites.

The duration measure did not provide as clear a picture for the 125 subjects as did the latency data, possibly because many of the rs were nonsignificant. For Trials 1 to 60, the two criteria conflicted. There was a suggestion that one, two, or more factors were significant ones. A one-factor resolution would have been similar to the solution obtained in the previous analysis with means. However, only $28.5 \%$ of the variance would have been accounted for. Two to five factors were extracted and rotated, using an Equimax procedure. Two factors provided the clearest solution, the primary site stimulation factor 
and the secondary site stimulation factor, as was the case with the latency measure. However, the plot of these two factors did not show as clear a separation of the trials on the different phases as did the latency data.

The two factors accounted for only $39.8 \%$ of the variance; this aspect is in sharp contrast to the $70.1 \%$ obtained with the latency measure. Also, the communalities for each trial were very low, varying from .061 to .626 . Many of these were below .300 . On the other hand, the latency communalities were sizable; except for the first six trials, these values tended to be .500 or greater, with many in the $.70 \mathrm{~s}$ and $.80 \mathrm{~s}$. These results indicate that the common factor variance was sizable for the latency trials, but nonexistent for the duration trials. For the latter measure, a great amount of specific factor variance and/or error variance was involved.

Three- to five-factor solutions did not improve on the two-factor resolution; instead, they tended to cloud the picture.

\section{One-Side Stimulation Only}

In order to highlight the sequential alternation results, data on 35 Wistar rats which had been stimulated only on one side over 10 phases of six convulsions each (Gaito \& Nobrega, 1978) were analyzed in the same fashion as that for alternation rats.

Means, standard deviations. The latency means for the 10 phases attained relative stability by Phase 2 and for the individual trials by Trial 6 (Table 4), in contrast to the alternation situation wherein relative stability was attained on Phases 3 and 4 for the two sides. The standard deviations were moderate from Phase 1 on in the data for both the phases and the individual trials.

Similar results occurred for duration data also (Table 5). Relative stability in means was attained by Phase 2 trials. Such results are similar to those obtained with alternating stimulation methods. The standard deviations were moderate from Phase 1 , except for Trial 1 and a few later trials (larger values usually occurred on the first trial of the day).

In criterion data, the Phase 1 mean was large and quite different from that for the remaining phases. The standard deviations followed this same pattern (Table 6).

Intercorrelations. ${ }^{2}$ With 35 subjects, the coefficient has to equal or exceed .330 to reject the null hypothesis of zero correlation. In phase data, the coefficients for the latency were sizable, all exceeding a value of .330. Most of the coefficients for the duration measure were significant also, although the magnitudes were not as great as those for latency data. On the other hand, most of the coefficients for the criterion measure were not significantly different from zero.

The intercorrelations for the 60 trials for the latency measures followed the same pattern as for the phase means; these coefficients were sizable and all significantly different from zero. Approximately half of the coefficients for the duration measure were significant ones, and the magnitudes tended to be much less than the latency coefficients.

Factor analyses. ${ }^{2}$ Although 35 subjects is a small number to use in a factor analysis, it was decided to conduct these analyses anyway, especially since size of sample is only one aspect that influences the outcome of a factor analysis. If the intercorrelation pattern is a clear one with such a small sample as $\mathbf{3 5}$, the factor analysis pattern should present clear and meaningful results.

Because few of the intercorrelations for criterion data were significant, a factor analysis was not conducted with these data. Such results would be meaningless. However, both the latency and duration data yielded a clear picture of a single general factor. The

Table 4

One-Side Stimulation: Means, Standard Deviations, and Linear Regression Coefficients (b) for Latency Measure

\begin{tabular}{|c|c|c|c|c|c|c|c|c|c|c|c|c|c|c|c|c|c|c|}
\hline \multicolumn{3}{|c|}{ Phases } & \multicolumn{16}{|c|}{ Trials } \\
\hline Phase & $\mathbf{M}$ & SD & Trial & $\mathbf{M}$ & SD & $\mathrm{b}$ & Trial & $\mathbf{M}$ & SD & $\mathrm{b}$ & Trial & M & SD & $\mathrm{b}$ & Trial & $\mathbf{M}$ & SD & b \\
\hline 1 & 8.3 & 4.1 & 1 & 13.0 & 6.6 & & 16 & 5.7 & 3.8 & & 31 & 7.9 & 6.2 & & 46 & 7.7 & 5.8 & \\
\hline 2 & 5.5 & 3.2 & 2 & 8.9 & 4.9 & & 17 & 5.4 & 4.0 & & 32 & 6.0 & 4.6 & & 47 & 5.8 & 4.6 & \\
\hline 3 & 5.7 & 3.5 & 3 & 7.6 & 4.6 & & 18 & 5.9 & 4.4 & .03 & 33 & 6.4 & 4.6 & & 48 & 6.8 & 5.4 & -.09 \\
\hline 4 & 5.9 & 3.8 & 4 & 7.8 & 5.3 & & 19 & 6.2 & 4.0 & & 34 & 7.5 & 5.8 & & 49 & 7.2 & 5.6 & \\
\hline 5 & 6.7 & 4.8 & 5 & 6.6 & 4.3 & & 20 & 5.4 & 3.5 & & 35 & 5.9 & 4.9 & & 50 & 6.4 & 5.2 & \\
\hline 6 & 6.6 & 4.7 & 6 & 5.9 & 4.1 & -1.21 & 21 & 6.2 & 4.1 & & 36 & 6.3 & 4.3 & -.21 & 51 & 6.9 & 5.5 & \\
\hline 7 & 6.8 & 4.9 & 7 & 5.9 & 3.8 & & 22 & 5.9 & 4.1 & & 37 & 7.8 & 5.9 & & 52 & 7.1 & 5.2 & \\
\hline 8 & 6.8 & 5.0 & 8 & 5.8 & 3.5 & & 23 & 6.0 & 5.5 & & 38 & 6.5 & 4.8 & & 53 & 5.9 & 3.9 & \\
\hline 9 & 6.6 & 4.6 & 9 & 5.8 & 3.7 & & 24 & 6.0 & 3.8 & .01 & 39 & 7.0 & 5.7 & & 54 & 6.0 & 4.3 & -.21 \\
\hline \multirow[t]{6}{*}{10} & 6.9 & 5.1 & 10 & 5.3 & 3.6 & & 25 & 6.5 & 5.0 & & 40 & 7.1 & 4.9 & & 55 & 8.3 & 7.6 & \\
\hline & & & 11 & 5.0 & 3.2 & & 26 & 6.0 & 4.1 & & 41 & 6.2 & 4.5 & & 56 & 6.4 & 5.0 & \\
\hline & & & 12 & 5.3 & 3.4 & -.17 & 27 & 7.0 & 4.9 & & 42 & 6.4 & 5.1 & -.22 & 57 & 6.3 & 4.7 & \\
\hline & & & 13 & 5.7 & 3.3 & & 28 & 7.1 & 4.9 & & 43 & 7.5 & 5.9 & & 58 & 8.2 & 6.7 & \\
\hline & & & 14 & 5.3 & 3.1 & & 29 & 6.6 & 5.9 & & 44 & 5.8 & 4.7 & & 59 & 6.1 & 4.9 & \\
\hline & & & 15 & 6.1 & 4.6 & & 30 & 7.0 & 5.3 & .13 & 45 & 7.2 & 5.6 & & 60 & 6.0 & 4.4 & -.30 \\
\hline
\end{tabular}


Table 5

One-Side Stimulation: Means, Standard Deviations, and Linear Regression Coefficients (b) for Duration Measure

\begin{tabular}{|c|c|c|c|c|c|c|c|c|c|c|c|c|c|c|c|c|c|c|}
\hline \multicolumn{3}{|c|}{ Phases } & \multicolumn{16}{|c|}{ Trials } \\
\hline Phase & $\mathbf{M}$ & SD & Trial & $\mathbf{M}$ & SD & b & Trial & $\mathbf{M}$ & $\mathrm{SD}$ & b & Trial & $\mathbf{M}$ & SD & $\mathrm{b}$ & Trial & $\mathbf{M}$ & SD & b \\
\hline 1 & 22.6 & 5.8 & 1 & 17.0 & 11.3 & & 16 & 26.3 & 6.7 & & 31 & 25.4 & 8.5 & & 46 & 26.1 & 7.1 & \\
\hline 2 & 26.1 & 4.6 & 2 & 21.1 & 8.3 & & 17 & 26.8 & 5.6 & & 32 & 29.5 & 7.8 & & 47 & 26.3 & 6.9 & \\
\hline 3 & 26.1 & 5.0 & 3 & 24.1 & 5.9 & & 18 & 27.4 & 7.2 & .59 & 33 & 30.0 & 8.3 & & 48 & 28.7 & 6.3 & -.24 \\
\hline 4 & 28.4 & 5.5 & 4 & 22.7 & 6.1 & & 19 & 29.7 & 9.2 & & 34 & 25.4 & 6.4 & & 49 & 28.5 & 9.3 & \\
\hline 5 & 28.2 & 5.4 & 5 & 26.0 & 7.2 & & 20 & 28.3 & 7.0 & & 35 & 28.0 & 6.4 & & 50 & 30.0 & 6.6 & \\
\hline 6 & 27.6 & 5.2 & 6 & 25.1 & 7.4 & 1.54 & 21 & 30.9 & 5.9 & & 36 & 27.7 & 6.6 & .07 & 51 & 31.3 & 7.8 & . \\
\hline 7 & 28.5 & 4.9 & 7 & 24.7 & 6.0 & & 22 & 27.2 & 8.3 & & 37 & 27.7 & 7.3 & & 52 & 26.0 & 8.5 & \\
\hline 8 & 28.0 & 4.5 & 8 & 25.7 & 7.7 & & 23 & 26.5 & 5.5 & & 38 & 29.8 & 5.4 & & 53 & 27.6 & 9.0 & \\
\hline 9 & 28.3 & 5.2 & 9 & 27.9 & 8.0 & & 24 & 28.2 & 6.8 & -.47 & 39 & 31.5 & 10.0 & & 54 & 26.8 & 6.5 & -.60 \\
\hline \multirow[t]{6}{*}{10} & 26.7 & 5.9 & 10 & 25.4 & 5.2 & & 25 & 27.2 & 8.4 & & 40 & 25.8 & 9.4 & & 55 & 26.5 & 10.3 & \\
\hline & & & 11 & 25.2 & 6.5 & & 26 & 28.7 & 6.7 & & 41 & 28.2 & 8.4 & & 56 & 26.9 & 7.4 & \\
\hline & & & 12 & 27.7 & 5.9 & .31 & 27 & 29.4 & 8.1 & & 42 & 28.4 & 7.3 & -.20 & 57 & 30.1 & 9.4 & \\
\hline & & & 13 & 24.0 & 7.2 & & 28 & 27.3 & 6.9 & & 43 & 27.0 & 10.3 & & 58 & 26.2 & 9.7 & \\
\hline & & & 14 & 25.5 & 7.9 & & 29 & 28.3 & 5.8 & & 44 & 31.0 & 7.3 & & 59 & 25.4 & 8.5 & \\
\hline & & & 15 & 26.6 & 8.0 & & 30 & 28.6 & 7.3 & .11 & 45 & 28.8 & 8.7 & & 60 & 25.1 & 9.5 & -.44 \\
\hline
\end{tabular}

proportion of variance extracted with this factor was .830 for the latency measure and .573 for the duration measure. Likewise, the magnitude of the communalities for each phase was greater for the former than for the latter.

The factor analyses for the 60 trials yielded a picture similar to that with phase means. Both the latency and duration measures had a single general factor, a greater proportion of variance was accounted for in the latency measure (.713 vs. .299), and the latency communalities were much greater than those for the duration data.

\section{GENERAL DISCUSSION}

Although the 125 subjects were stimulated with near-threshold intensities for each side in contrast to the use of the same intensities for both sides with the 139 subjects of the previous research, the results in this study provide an effective replication of those of the previous studies (Gaito et al., 1977; Gaito \& Gaito, 1979) in that parallel results occurred. The findings of these studies are:

(1) The means for the first two phases in the latency and criterion measures were the greatest, and relative stability occurred during Phases 3 and 4.

(2) The latency means for the secondary site were greater than those for the primary site for all phases. This result produced a primary oscillation pattern and a saw-tooth curve over phases.

(3) The duration means for the phases were different from those for latency and criterion data in two respects: (a) lowest values occurred on Phase 1, and (b) relative stability was present during Phase 2.

(4) The means for the 60 trials tended to parallel the results with phase means. In latency data, the first trial in all phases tended to be the greatest. In duration data, the first tended to give the lowest value.

(5) Intercorrelations for the latency measure showed a pronounced "low-high" pattern over phases, and criterion means showed a similar, but less pronounced, pattern. Duration data gave a more homogeneous matrix of values.

(6) The factor analysis pattern for the latency measure was a clear one: two well-separated factors emerged in all cases (primary site stimulation factor, secondary site stimulation factor), with slight correlation occurring between these factors. This correlation aspect is probably an indication of the positive transfer effects from primary to secondary sites.

(7) The duration measure could be represented by a single factor in phase means data, but a two-factor solution provided a better result both in phase data and in the data from individual trials. However, the two factors were not as clearly separated as in the latency case.

(8) The criterion phase data gave a two-factor resolution also, but not a clear separation result.

(9) The proportions of variance accounted for by the factors extracted and the communalities for each phase (or each trial) were greater for the latency measure than for the duration and criterion measures.

(10) These results for sequential alternation of stimulation for primary and secondary sites contrasted sharply with those for data when stimulation occurred only on one side. The data in the latter case were

Table 6

One-Side Stimulation: Means (M) and Ștandard Deviations (SD) for Criterion Measure for 10 Phases

\begin{tabular}{lrrrrrrrrrr}
\hline & 1 & 2 & 3 & 4 & 5 & 6 & 7 & 8 & 9 & 10 \\
\hline $\mathrm{M}$ & 18.3 & 6.1 & 6.5 & 6.1 & 6.2 & 6.1 & 6.6 & 6.3 & 6.2 & 6.5 \\
$\mathrm{SD}$ & 8.1 & .2 & 1.1 & .4 & .5 & .2 & 3.0 & 1.4 & .9 & 1.6 \\
\hline
\end{tabular}


Table 7

Factors or Mechanisms Present During Kindling Using the Latency Measure

\begin{tabular}{lll}
\hline & $\begin{array}{c}\text { Goddard et al. (1969) } \\
\text { McIntyre-Goddard (1973) }\end{array}$ & Factor Analyses \\
\hline $\begin{array}{l}\text { Sequential } \\
\text { Alternation } \\
\text { (2 sides) }\end{array}$ & $\begin{array}{l}\text { Neurological trace with } \\
\text { aftereffect (primary site) }\end{array}$ & $\begin{array}{l}\text { primary site } \\
\text { stimulation factor }\end{array}$ \\
& $\begin{array}{l}\text { Neurological trace with } \\
\text { aftereffect (secondary site) }\end{array}$ & $\begin{array}{l}\text { seconday site } \\
\text { stimulation factor }\end{array}$ \\
$\begin{array}{lll}\text { One-Side } \\
\text { Stimulation }\end{array}$ & $\begin{array}{l}\text { Neurological trace with } \\
\text { aftereffect (primary site) }\end{array}$ & $\begin{array}{l}\text { primary site } \\
\text { stimulation factor }\end{array}$ \\
\hline
\end{tabular}

more homogeneous. A single factor emerged for each of the three measures in sharp contrast to the two factors which are present in the alternation case.

These factor analysis results, and the previous ones, appear to support the two mechanisms of Goddard et al. (1969) and McIntyre and Goddard (1973) concerning brain changes during kindling. Their first factor was a positive transfer one of a long-term nature, involving brain circuitry changes in both sites, and the "aftereffect"' (second factor) was a less durable one of a negative transfer nature. However, the "aftereffect" is involved in convulsions from stimulation of both the primary and secondary sites. Thus, the two factors can be viewed as (1) a primary site factor of a neurological trace nature with a residual "aftereffect," and (2) a secondary site factor of a neurological trace nature with a residual "aftereffect." The two factors of neurological trace and aftereffect are confounded in our behavioral data (Table 7). Thus, the factor analyses are showing the operation of these two in each of the two factors, one for the primary site and the other for the secondary site. The development and stabilization of the neurological mechanisms involved are indicated in behavior during the early phases by the decrease in latency, decrease in number of trials to achieve the criterion of six $\mathrm{CC}$, and the increase in duration of convulsion.

The consistency of these results and the Goddard et al. and McIntyre and Goddard interpretation of brain function during kindling is illustrated most appropriately by the latency data (Table 7). These data have tended to provide the most definite results, both in each individual experiment and in each of the factor analyses attempted.

The results of the previous study applied to subjects which were exposed to different stimulation conditions, e.g., different numbers of trials per phase, different number of convulsions per phase, etc. Likewise, the subjects in this study had been exposed to different conditions in each of the separate experiments. Furthermore, these 125 subjects had been ex- posed to near-threshold stimulation intensities for each side, whereas the 139 subjects of the previous study had the same intensities for both sides. Thus, one would expect some degree of caution in the interpretation of results under such conditions. However, the fact that similar results occurred in both studies should provide one with greater confidence in these results. Thus, the results over both samples would appear to provide valuable norms for brain stimulation research under various conditions.

\section{REFERENCES}

Garto, J. The kindling effect as a model of epilepsy. Psychological Bulletin, 1976, 83, 1097-1109. (a)

GaITo, J. An oscillation effect during sequential alternations of unilateral amygdaloid stimulations within the kindling paradigm. Psychological Psychology, 1976, 4, 303-306. (b)

GaITo, J. The oscillation effect at near-threshold intensities during sequential alternation of unilateral amygdaloid stimulation. Bulletin of the Psychonomic Society, 1977, 10, 145-148.

Gaito, J., \& Gaito, S. T. Interanimal negative transfer of the kindling effect. Physiological Psychology, 1974, 2, 379-382.

Gaito, J., \& Gaito, S. T. Statistical analyses of oscillationeffect data within the kindling paradigm. Bulletin of the Psychonomic Society, 1979, 14, 385-388.

Gaito, J., \& Nobrega, J. N. Random oscillation patterns with stimulation of a single brain site. Bulletin of the Psychonomic Society, 1978, 11, 65-67.

Gaito, J., Gaito, S. T., \& Nobrega, J. N. A factor analysis of data from 10 phases of sequential alternations of amygdaloid stimulation within the kindling paradigm. Physiological Psychology, 1977, 5, 300-310.

Gaito, J., Nobrega, J. N., \& Gaito, S. T. Statistical evaluation of several aspects concerning the oscillation effect. Physiological Psychology, 1978, 6, 209-214.

Goddard, G. V., McIntyre, D. C., \& Leech, C. K. A permanent change in brain function resulting from daily electrical stimulation. Experimental Neurology, 1969, 25, 295-330.

Gorsuch, R. L. Factor analysis. Philadelphia: Saunders, 1974.

McInTyre, D. C., \& Goddard, G. V. Transfer, interference and spontaneous recovery of convulsions kindled from the rat amygdala. Electroencephalography and Clinical Neurophysiology, 1973, 35, 533-543.

RACINE, R. J. Modification of seizure actvity by electrical stimulation: I After-discharge threshold. Electroencephalography and Clinical Neurophysiology, 1972, 32, 269-279.

Racine, R. Kindling: The first decade. Neurosurgery, 1978, 3, 234-252.

WAdA, J. A., \& SAto, M. The generalized convulsive seizure state induced by daily electrical stimulation of the amygdala in split brain cats. Epilepsia, 1975, 16, 417-430.

\section{NOTES}

1. The curves for latency, criterion, and duration data are similar to those of Figures 2, 3, and 4, respectively, in Gaito, Nobrega, and Gaito (1978).

2. All intercorrelations and factor analysis data may be obtained by writing to the first author.

3. Communality is the sum of squared loadings on each factor, i.e., the proportion of common factor variance.

(Received for publication May 23, 1979; revision accepted October $15,1979$. 